\title{
How to diagnose neuropathy in diabetes mellitus?
}

\author{
Abdulkadir Koçer \\ Department of Neurology, Istanbul Medeniyet University School of Medicine, Istanbul, Turkey
}

\begin{abstract}
Diabetic neuropathy is a common problem and can present various clinical presentations such as cranial neuropathy, radiculopathy, plexopathy, mononeuropathy, polyneuropathy and autonomic neuropathy. Clinical evaluation and use of scoring systems for the evaluation of diabetic polyneuropathy is an important step to get correct diagnosis. New informations about the diagnosis of diabetic neuropathy continue to emerge, which will lead to correct diagnosis and treatment as well. Diabetic length-dependent sensorimotor polyneuropathy (DSPN) and carpal tunnel syndrome are the most common seen problems. While acute and painful situations with motor weakness are mostly transient, sensory fibres are predominantly involved in chronic ones. Nerve conduction studies are needed to confirm diagnosis of any type of diabetic neuropathy, but they are normal in cases with small fiber involvement. The early diagnosis is crucial because it is well-known that subclinical diabetic neuropathy may be reversed or significantly improved with diabetes control. Because neuropathy at the stage in which only small fibers are affected can be reversed, it is important to diagnose DSPN in these stages. Skin biopsy taken from the dermatomal area of sural nerve, laser-doppler-imager flare technique, corneal confocal microscopy are used to assess small fibre dysfunction. The aim of the present review was to evaluate evidence-based diagnosis for any type of neuropathy seen in the patients with diabetes mellitus.
\end{abstract}

Eur Res J 2018;4(2):55-69

Keywords: length-dependent sensorimotor polyneuropathy, small fiber, nerve conduction studies, neuropathic pain, scoring systems, diabetes mellitus

\section{Introduction}

Neuropathy developing secondary to diabetes is not only a late complication but also can develop at any time during the course of the disease. It should be evaluated carefully because neuropathy symptoms are associated with poor quality of life [1]. The neuropathies developing in patients with diabetes are heterogeneous and may be separated into generalized and focal/multifocal varieties [2-4]. Onset of symptoms is also variable and may be acute, subacute, or chronic in forms. Symmetric or generalized neuropathies usually present as chronic neuropathies and asymmetric ones present as acute forms with more prominent pain [2-4]. Diabetic neuropathy diagnosis needs exclusion of other causes for peripheral neuropathy and and it is also increasingly recognized in patients with prediabetes who are at high risk of 
developing diabetes mellitus (DM). As it was seen Figure.1, there are different types of neuropathy seen in diabetes. The most commonly seen and typical form is a chronic, symmetrical, length-dependent sensorimotor polyneuropathy and is thought to be the most common variety [5]. The second most commonly problem seen in the patients with diabetes is carpal tunnel syndrome [6]. Distal symmetric polyneuropathy (DSPN) may be associated with retinopathy and nephropathy for which total hyperglycemic exposure is perhaps the most important risk covariate [7, 8]. Autonomic dysfunction and neuropathic pain may develop over time as well [7]. Chronic inflammatory demyelinating polyradiculopathy (CIDP) is another problem which may be seen in diabetic patients. It is well known that similar patterns of DSPN may occur in patients without diabetes, but with other toxic or metabolic causes [7]. Although many neuropathic problems were reported in the literature, there is not any diagnostic algorithm. The presence of atypical polyneuropathies, which are different from DSPN in several important features, is another problem to be evaluated. In this report, we will test to review the old advances and present some practical clues about the diagnosis of neuropathies in patients with diabetes mellitus.

\section{Prevalence of any Case of Neuropathy in Diabetes}

Both type I and type II diabetes are the most usual cases of neuropathy worldwide. The prevalence of neuropathy changes from $50 \%$ to $90 \%$, depending on the criteria and methods used [5, 9-11]. Almost one and half of the diabetics had classical symmetric polyneuropathy, $25 \%$ had carpal tunnel syndrome,

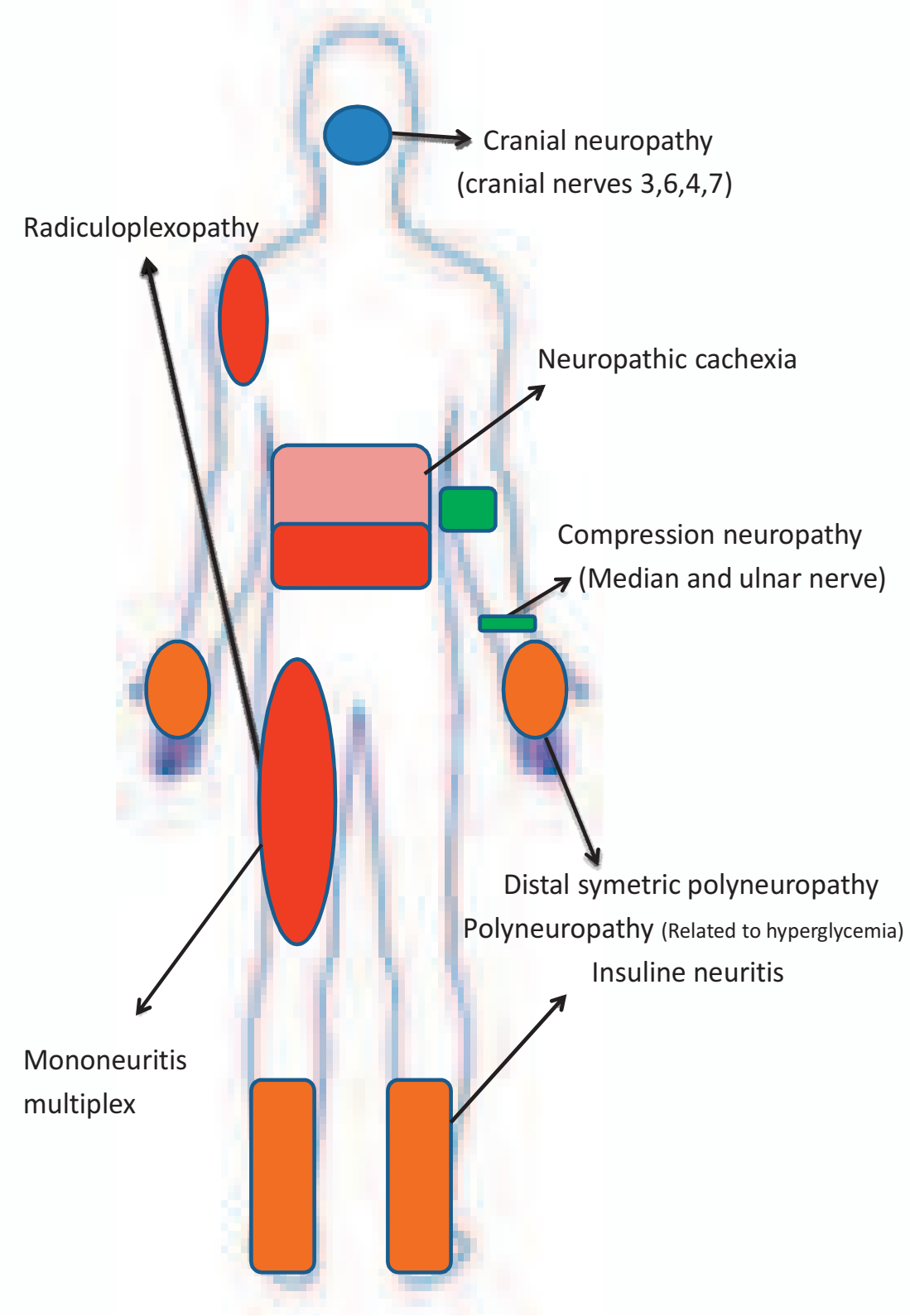

Figure 1. Types of neuropathy seen in diabetes. 
about $5 \%$ had autonomic neuropathy and $1 \%$ had asymmetric proximal neuropathy [6]. The occurrence of any type of neuropathy correlates with the duration of diabetes, poor glycemic control and with the presence of retinopathy and nephropathy [5]. In patients with neuropathy associated with impaired glucose tolerance, controlled diet and exercise may lead to re-innervation [12]. Findings from subclinical neuropathy studies suggest that intensive control of glycemia may result in improved nerve conduction
[13]. Neuropathic pain is a very popular and an important problem causing discomfort in patients with diabetes. Neuropathic pain is a pain arising as a direct consequence of a lesion or disease affecting the somatosensory system [14]. Bilateral thalamus, spinal cord, left parietal lobe and subcortical located deep nuclei are involved in pain generation in diabetes patients [15]. The etiology of neuropathic pain is broad, and diabetes is a significant and widely seen cause $[4,14,15]$. Neuropathic pain is also one of the

Table1. The diagnostic approaches in the patients with suspicion of diabetic neuropathy.

DIAGNOSTIC PROCEDURES

Neurological Examination

Diabetic neuropathy examination (DNE) score

Diabetic symptom score (DNS)

Nerve conduction studies

\section{Screening laboratory tests}

Two-hour glucose tolerance testing

Vibration sensation

(Halux of great toe)

The Semmes-Weinstein 1-10 gram monofilament examination (SWME) Genetic testing

\section{Neuroimaging (MRI)}

Skin biopsy from the dermatomal area of sural nerve

Laser-Doppler-imager flare technique

\section{Neuropathic pain assessment}

Corneal confocal microscopy

\section{WHEN THE PROCEDURE SHOULD BE USED}

1- Sensory examination: response to light touch, temperature, painful stimulus, vibration and proprioception.

2- Motor examination: testing muscle tone and strength, reflexes and coordination.

3- Autonomic findings: examing changes in colour, temperature, sweating and swelling.

1- In any type of diabetic polyneuropathy

1- In distal symetric polyneuropathy (DSPN)

4- In atypical cases with superimposed nerve entrapments or inflammatory demyelinating neuropathy such as CIDP

5- In neuropathy cases with asymmetrical symptoms

6- In cases with involvement of the upper limbs

7- In any period of DSPN (To confirm diagnosis)

1- Serum glucose, HbA1C ve glucose tolerance test

2- For differential diagnosis in DSPN cases Blood sugar, blood count, ESR, anti-HIV Ab, anti-Lyme $\mathrm{Ab}$, Liver and renal function tests, serum vitamin $\mathrm{B}_{12}$, serum protein elctrophoresis, vasculitis profile, paraneoplastic markers, thyroid function tests

1- In patients with an otherwise idiopathic neuropathy

2- In patients with chronic idiopathic axonal polyneuropathy

1- In early diagnosis of neuropathy

2- In large-fiber involved cases in diabetes

3- In all cases of other types of polyneuropathy

1- In early diagnosis of neuropathy

2- In large-fiber involved cases in diabetes

1- In patients only with a history of hereditary neuropathy and cryptogenic polyneuropathy who exhibit a hereditary neuropathy phenotype

1- In occulomotor nerve involved cases with pupillary involvement or absence of pain

2- In radiculopathy or plexopathy cases

1- In early diagnosis of DSPN to assess small-fiber function

2- In evaluation of NP development risk

1- In early and reversible stage of DSPN to assess small-fiber function (in whom results of conventional quantitative sensory tests and skin biopsy results are normal)

1- In any polyneuropathy patients to identify and quantify neuropathy

1- In early diagnosis of DSPN to assess small-fiber function

2- In evaluation of severety of neuropathy and NP development risk 
Table 2. Toronto clinical neuropathy scoring system for the evaluation of diabetic polyneuropathy.

\begin{tabular}{llc}
\hline Symptom scores & Reflex scores & Sensory test scores \\
\hline Foot & Knee reflexes & Pinprick \\
Pain & Ankle reflexes & Temperature \\
Numbness & & Light touch \\
Tingling & & Vibration \\
Weakness & & Position \\
& \\
Ataxia & & \\
Upper-limb symptoms & & \\
\hline
\end{tabular}

Recording of scores (Symptom scores: present $=1$; absent $=0$. Reflex scores: absent $=2$; reduced $=1$, normal $=$ 0 . Sensory test score: abnormal $=1$. normal $=0$ ).

most common presentations not only in diabetes, but also in impaired glucose tolerance and impaired fasting glucose $[4,12,14]$. While the relationship between diabetes and neuropathic pain is well documented, the occurrence rate of neuropathic pain is still underdiagnosed and difficult to quantify [11, 15]. Neuropathic pain occurs in $7.5 \%$ to $35 \%$ of patients with DM $[11,15]$.

\section{Neurological Examination and Diagnostic Procedures}

Any toxic, metabolic or systemic inflammatory diseases resulting in distal type axonal neuropathies, small fiber neuropathies, demyelinating neuropathy especially CIDP, multifocal neuropathies and radiculopathies or plexopathies may mimic neuropathy seen in diabetes. Neurologic examination focuses on the recognition of clinical signs of neuropathy. Patients are asked to report whether they experienced gait problems (difficulty in walking or climbing, ataxia), thermal allodynia (e.g. When they have a bath) and/or pain in the feet. For evaluation of diabetic neuropathy, neurological examination should include cranial nerve examination, assessment of muscle force, sensations of pinprick, touch, temperature, joint position and vibration. It is also tried to identify the presence and distribution (e.g. length or non-length dependent) of any type of sensory loss and pain. At the second step, symptoms and signs of dysautonomy are recorded. Dysautonomic symptoms (e.g. pupil abnormalities, impotence, impaired bladder function, constipation or diarrhea, early satiety and gastric fullness, abnormal sweating, flushing, skin discoloration, xerostomia and xerophthalmia, orthostatic hypotension) are recorded. The examination clicks, and diagnostic processes are summarized below and some diagnostic clues are presented on Table 1. They are also detailed in the following section named as "classification and diagnostic work-up in diabetic neuropathies".

In the algorithm, a patient with neuropathy should be assessed in order mentioned below.

\section{Evaluation of neuropathic symptoms and} signs:

The patients are assessed by the Toronto Clinical Scoring System for Diabetic Polyneuropathy, a validated instrument reflecting the presence and severity of DSPN as measured by sural nerve morphology and electrophysiology [16]. Sensory testing was performed on the first toe. Total scores range from normal $=0$ to a maximum of 19 . Mild DSPN was defined from a score of 5 onwards, and severe neuropathy as a score of 10 (see Table 2). Diabetic neuropathy examination (DNE) score, which is a modification of the Neuropathy Disability Score of Dyck is another scoring and identification way of neuropathy in which neurological examination was carried out and the neurological signs were recorded [17]. The DNE consists of eight items (Extension of the knee and dorsiflexion of the foot, Sensitivity to pinpricks at index finger and great toe, Ankle reflex, Sensitivity to touch, Vibration perception and Sensitivity to joint position). More than three abnormal results in these exams indicate presence of polyneuropathy. A third way of evaluation DSPN is to record diabetic symptom score (DNS). The DNS score has the following items: (1) unsteadiness in walking, (2) pain, burning or aching inthe legs or feet, (3) prickling sensations in the legs or feet, and (4) numbness in legs or feet. Presence is scored 1 , absence 0 , maximum score 4 points. The DNS is valid in cases of diabetic polyneuropathy, and is fast and easy to perform in clinical practice [18].

\section{Examing muscle power and deep tendon}


reflexes:

Motor and reflex exams are more important in the evaluation of other neuropathies such as radiculo/plexopathies developed secondary to diabetes. Although ankle reflex is a powerful screening tool, a combination of ankle reflex and vibration sense has superior sensitivity and specificity compared with either of them done alone for the detection of DSPN in clinical settings [19]. Muscle strength was graded using the Medical Research Council (MRC) score. Positive Romberg's sign and gait abnormalities were recorded. Ankle reflexes are obtained at both ankles. The examiner gently dorsiflex the foot and strike the achilles tendon briskly with the reflex hammer. Deep tendon reflexes were graded as normal, decreased (with reinforcement) or absent. It may be normal to have reduced or absent ankle reflexes in some elderly people, although the frequency and significance of this is disputed [20,21]. It should be also remembered that the isolated loss of a reflex can point to a radiculopathy affecting that segment e.g. loss of ankle jerk if there is an S1 disc prolapse.

\section{Quantitative sensory testing:}

It is an important title meaning an objective index of neurologic functional status in the assessment of neuropathy in diabetes. The normal ranges of these tests vary systematically according to the age of the patients. These tests require the patient to be alert and cooperative for reliable results because quantitative sensory testing is a psychophysical test and lacks the objectivity of nerve conduction studies. Another problem is the weak correlation between these tests indicating the need to apply more than one test in screening for diabetic neuropathy [22].

a) Touch and pain sensation: Thouch sensation examination is performed by using cotton gauze (to test light touch and dynamic mechanic allodynia). Disposable safety needle (to test hypoalgesia, pinprick hyperalgesia) is applied for evaluation of pain sensation. Sensory loss and positive sensory signals (evoked and spontaneous pain, paresthesias) are recorded for both types of sensory modalities. Superficial pain sensation may be conducted using a sterile Neurotip (Owen Mumford, Oxford, U.K.) and it is applied at the eponychium of the first toe and it is repeated in an arrhythmic manner to the three sites described for the SWME at next paragraph and one side for the Neurotip. The superficial pain threshold is defined as the total number of times the application of the pain sensation is not perceived, with scores varying from 0 to $8[23,24]$.

b) The Semmes-Weinstein monofilament examination ( $S W M E)$ : It is a noninvasive, lowcost, rapid (takes less time, $<5$ minutes), and practical test often used in clinical testing of pressure sense $[25,26]$. The 5.07/10 g Semmes Weinstein monofilament assesses the integrity of Merkel touch domes and Meissner's corpuscles and their associated large diameter fibers [27]. The variability in the methodology of SWME (weight of the filament, the number of sites, location of sites, and the diagnostic threshold) limits the effectiveness of the test as a diagnostic tool. In general, nine plantar sites (distal great toe, third toe, and fifth toe; first, third, and fifth metatarsal heads; medial foot, lateral foot, and heel) and one dorsal site were examined. The monofilaments are applied to the test site perpendicularly until they bend for about one second. Patients are instructed to say "yes" each time they sense the monofilament on their foot. If patients fail to sense the monofilament after it twists, the test site is thought to be insensate $[28,26]$. It was repeated four times on both feet in an arrhythmic manner. The SWME threshold was defined as the total number of times the application of $10-\mathrm{g}$ monofilament was not perceived by the patient, and it varies according to the number of placed. Patients unable to detect one or more sites should be classified as at risk in order to maximize sensitivity [26]. The quantitative analysis of the 16 studies with sufficient data revealed that SWME is both fairly sensitive and highly specific when compared with the gold standard of NCS. While the use of 5.07/10 g SWME is widespread and generally accepted, data show biothesiometry and the use of finer monofilaments ( $1 \mathrm{~g})$ may improve detection rates of abnormality [29-31].

c) Vibration sense: Examing vibration sensation reflects large fiber involvement and it is performed by using $128 \mathrm{~Hz}$ Rydel-Seiffer tuning fork (vibration at the first metatarsal joints, ankles, knees, first metacarpal joints, elbows). For the best result, a 128$\mathrm{Hz}$ tuning fork is applied to the halux bilaterally situated at the lateral side of the first toe [32]. The onoff method or the timed method is used in testing of vibration. It is recorded whatever the patient reports about the perception of both the start of the vibration sensation and the cessation of vibration. The testing is conducted twice on each toe, and correct responses are recorded [23, 33, 34]. The vibration can be felt for 10 to 15 seconds. Patients are asked initially, and after 5 seconds, whether they perceive vibration. Patients who felt vibration both initially and after 5 seconds are 
scored as normal. Vibration not perceived at all is scored as absent, and vibration perceived initially but not at 5 seconds is scored as abnormal. The vibration test is reproducible and accurate test. It provides a quantitative assessment of DSPN and can document severe neuropathy, even in the presence of a normal result with the 10-g monofilament test [33]. The risk of foot ulcers is associated with diminished vibratory sensation and can be detected earlier and more accurately with the great toe vibration test. It is accepted that great toe vibration test should replace the 10-g monofilament test as the recommended technique for detection of DSPN [33].

d) Thermal sensory testing: Quantitatively testing of warm and cold is one of the tests currently being used for early detection of diabetic neuropathy such e. g. DSPN (This test is performed by using glass tubes filled with cold and hot water (thermal sensation, allodynia test, aftersensation test). Sensory loss and positive sensory signs (evoked and spontaneous pain, paresthesias) are recorded [22, 35]. Nowadays, pain and temperature sensation thresholds are measured by some devices for the evaluation of small nerve fiber dysfunction [36].

e) Pain-related evoked potentials: It is elicited by a nociceptive electrical stimulation of the skin and may contribute to the early detection of diabetic sensory neuropathy [37]. This method is one of the easiest and most reliable of the neurophysiological methods for assessing function of nociceptive pathways (mainly A delta fibers) [25].

f) Position sense: Sense of movement and position of toes and hand fingers were evaluated. Position sense was assessed at the interphalangeal joint of each great toe for a position change. The toe was held at both sides of proximal phalanx with one hand while using the other hand to move the distal phalanx up or down. After three trials of exam for each foot, the patient was asked to choose "up or down?" and the three responses per foot were scored as correct or incorrect [38].

\section{Electromyography:}

Nerve conduction studies (NCSs) are the most frequently used diagnostic tool for evaluation of any case of neuropathy in the patients with diabetes.

\section{Corneal confocal microscopy:}

It is a new sensitive non-invasive examination, which may be used to detect early small fiber nerve damage in patients with small nerve fiber neuropathy. Corneal nerve parameters are assessed and it allows quantification of corneal nerve morphology. Cornea nerve damage correlates with the severity of pain and autonomic neuropathy $[39,40]$.

\section{Skin biopsy:}

Nowadays it is included in the diagnostic work-up of patients with suspected small-fiber neuropathy [41]. This technique is used to show that small fibers degenerate early in the course of neuropathies associated with diabetes and it confirms the diagnosis when clinical and neurophysiologic examinations are not informative for diabetic neuropathy $[42,43]$. A skin biopsy is also important to say about pain. Information from the literature suggest that a more serious loss of intraepidermal nerve fiber density increases the hazard to develop neuropathic pain, whereas intraepidermal nerve fiber density regeneration can be linked with a reduction in pain intensity [44]. Skin biopsy shows a diagnostic efficiency of $88.4 \%$, clinical examination of $54.6 \%$ and quantitative sensory tests about $46.9 \%$ in patients with small fiber neuropathy $[22,35,41]$.

\section{Laser-Doppler-imager (LDI) flare technique:}

LDI flare may represent a novel method for detecting early small-nerve-fiber changes in people with diabetes. The LDI flare easily detects small-fiber dysfunction before the occurrence of potentially irreversible structural loss of nerve fibers and can demonstrate abnormal C-fiber function in subjects with symptomatic DSPN in whom the results of conventional quantitative sensory tests are normal and in whom there is no significant reduction in nerve fiber density NFD [24]. In this non-invasive technique, axon reflex mediated vasodilatation in response to skin heating is measured and $\mathrm{C}$-fiber dysfunction is detected in type 2 diabetes before small-fiber neuropathy can be discovered by other currently available noninvasive methods [24, 45]. Skin proximal to the first and second metatarsal heads on the dorsum is heated with a circular skin heater (diameter $1.0 \mathrm{~cm}$; Moor Instruments) to $44^{\circ} \mathrm{C}$ for $20 \mathrm{~min}$. An area of surrounding the heated skin was scanned with the LDI immediately after careful removal of the heater probe $[24,45]$. On the flux image, the region of interest demarcated by the edge of the flare is drawn, and the area of the LDI flare is calculated and expressed in square centimeters [24].

\section{Neuropathic pain assessment:}

Although only some patients with nerve lesions develop neuropathic pain, it is a significant issue 
because neuropathic pain is associated with poor quality of life and in that respect is no recognized objective gold standard for assessing neuropathic pain. Clinical assessment and diagnosis of neuropathic pain, a clinical history of pain and sensory exam are established to confirm nervous system involvement. Standardized screening tools which are often applied to distinguish and quantify neuropathy provide a full clinical record for follow-up of the patients with any case of neuropathy [46]. The diagnostic accuracy of neuropathic pain screening and assessment tools varies across patient populations. The DN4 may be more universally applicable than other creatures (e.g. The Leeds assessment of neuropathic symptoms and signs, PD-Q, and Neuropathic Pain Questionnaire) in general [47]. In DN4 scoring system, the presence of neuropathy symptoms (1 - Burning, 2 - Painful cold, 3 - Electric shocks, 4 - Tingling, 5.- Pins and needles, 6 - Numbness, 7 - Itching) and examination findings (1 - Hypoesthesia to touch, 2 - Hypoesthesia to prick, 3 - Allodynia by brushing) are evaluated. The total score is calculated as the sum of the 10 items and the cutoff value for the diagnosis of neuropathic pain is a total score of $4 / 10$.

\section{Classification and Diagnostic Work-up in Diabetic Neuropathies}

\section{A. Chronic symmetric neuropathies}

\section{Diabetic sensoriomotor polyneuropathy}

The most common complication is peripheral neuropathy and distal symmetric polyneuropathy (DSPN) occurring in up to $50 \%$ of patients is the most common presentation of neuropathy in diabetes [4850]. Chronic inflammatory demyelinating neuropathy which has similar sensory findings as seen in DSPN of diabetes is also more common in diabetic than in non-diabetic patients [51]. Typical DSPN is defined as a "chronic, symmetrical, length dependent sensorimotor polyneuropathy" $[50,52]$. It is a commonly seen polyneuropathy with small and large fibre sensory, motor and autonomic involvement in various combinations. Diagnosis of DSPN is primarily clinical and needs a good history taking and neurological examination $[50,52]$. A minimum of two abnormalities (from symptoms, signs, nerve conduction abnormalities, quantitative sensory tests, or quantitative autonomic tests) is needed in diagnosis [53]. In other words, documenting an abnormal sensory and motor examination of feet and great toe, and Aschille tendon reflex is sufficient to diagnose neuropathy with appropriate symptoms in a diabetic patient. Because of this reason, the use of monofilaments to assess touch-pressure sensation should not be forgotten as well as the Rydell-Seifert semi-quantitative tuning fork $[54,55]$. New diagnostic techniques (including measurement of nerve fiber density using corneal confocal microscopy, and nociceptive evoked potentials) may contribute to the diagnostic work-up [53-56]. In the examination of the most serious complications such as foot ulcers, Charcot foot abnormalities and small injuries, they are checked visually [56]. To assess peripheral vascular disease as a possible cause of foot problems, peripheral pulses are checked as well.

Symptoms and signs: Sensory symptoms are more prominent, and the neuropathy symptoms start in the feet and spread proximally in a stocking glove distribution with increasing duration or severity of diabetes [57]. Both "positive" (tingling, burning, and other abnormal sensations) and "negative" (sensory loss, weakness, numbness, and unsteady gait) sensory symptoms may be seen $[50,52,57,58]$. Small fibers are firstly effected and manifests with the impairment of pain, temperature and autonomic functions. Patients may report superficial burning pain or deep aching pain, tingling and numbness in their toes $[11,59]$. They feel that their feet are persistently cold. Progression of sensory symptoms predisposes the patients to falls, development of foot ulcers and decreased quality of life [58]. Upwards to $50 \%$ of patients may experience neuropathic pain symptoms worse at night and disturb sleep [60]. What a pity, as up to $50 \%$ of the patients may be asymptomatic, a diagnosis may simply be made on examination and the patient presents with a painless foot ulcer in some cases $[56,60]$. Small fiber neuropathies may be experienced even in the patients with impaired glucose tolerance and motor and reflex tests are normal during that stage $[11,48,59]$. Subsequently, the large nerve fibers are damaged and then gait disturbance due to impaired proprioception, mild distal weakness such as weakness of big toe and autonomic symptoms (pallor alternating with rubor, cyanosis, and mottling) may be seen $[11,57,58]$. Examination of the lower limb reveals distally located signs such as loss of pinprick, temperature, touch and vibration sense with reduced or absent ankle jerks and loss of great toe position sensation $[60,64]$. Distal weakness occurs only in the most severe instances, so testing of the Achilles tendon reflex often indicating DSPN even in mild or asymptomatic cases is significant. In a typical DSPN 
case, monophasic or fluctuating course and asymmetric or proximal symptoms as well as motor involvement may be seen. Acute neuropathic pain localized distally is another atypical characteristic of DSPN cases and pain may come in even prediabetes cases [61-63]. Any two of the following: neuropathy symptoms, decreased distal sensation, or decreased/absent ankle reflexes make DSPN diagnosis probable. For a definite diagnosis, it should be confirmed by abnormal nerve conduction study [64].

Differential diagnosis: Any variation of clinical findings such as acute, asymmetric, proximal, or motor involvement suggests an atypical neuropathy. In cases with sensory ataxia or profound distal upper and lower extremity weakness, an evaluation for other causes of neuropathy i.e. CIDP is recommended [65]. It should be also remembered that progression of DSPN is usually quite slow except the unusual cases of severe sensory and autonomic neuropathy that can occur in the first several months after the onset of Type 1 diabetes $[65,66]$. The severe proprioceptive problem occurs when there is prominent large fiber involvement. These patients develop sensory ataxia and autonomic manifestations with impotence, bladder atony, and pupillary changes and thus have been called the pseudotabetic form of diabetic neuropathy. The presence of proprioception deficits and ataxia should lead to a search for other possible etiologies (syphilis, B12 deficiency, paraneoplastic or Sjogrens syndrome sensory neuropathy, CIDP) $[66,67]$. Nevertheless, it is thought that the pseudosyringomyelic, pseudotabetic, and the early onset neuropathy described by Said et al. [66], all are severe variants of diabetic DSPN and probably not distinct forms of neuropathy [65-67]. Finding electrophysiologic changes that fulfill demyelinating criteria superimposed on axonal degeneration in a diabetic should be assessed with care. Likewise progressive polyradiculoneuropathy with a presentation similar to CIDP in diabetes may be seen, CIDP can develop in diabetic patients, too [67-69]. The clinical presentation of progressive poly-radiculoneuropathy is dominated by painful progressive motor weakness, with or without exacerbation of sensory symptoms. The weakness involves all limbs, but is often asymmetric. These symptoms and signs are important in differential diagnosis from classical DSPN. Electrophysiology of polyradiculoneuropathy may be predominantly axonal similar to DSPN, and CSF may show increased protein similar to CIDP cases [69]. Becuase nerve conduction studies and nerve biopsy are not helpfull in differential diagnosis, the clinical features of gradually progressive, usually painless, proximal and distal symmetric weakness and numbness in the arms and legs should be sufficient to distinguish CIDP from the typical symmetric and asymmetric diabetic neuropathies [65].

Screening tools: Utah Early Neuropathy Scale examing great toe dorsiflexion, pin sensation, great toe vibration and joint position, allodynia, and deep tendon reflexes is a sensitive and reproducible clinical measure of sensory and small-fiber nerve damage and may be useful in early diagnosis of diabetic neuropathy [70]. On the other hand, several examination scales focusing on large-fiber function have been risen to establish DSPN diagnosis. The Michigan Neuropathy Screening Instrument (MNSI) is a good screening tool for diabetic neuropathy and the Michigan Diabetic Neuropathy Score (MDNS) having higher concordance with nerve conduction studies provides a simple support to confirm this diagnosis $[71,72]$. In the evaluation of neuropathic pain in diabetes, DN4 questionnaire or LANSS easily identifies potential patients with neuropathy, particularly by non-specialists, but it should be remembered that they cannot replace careful clinical judgement [73].

Electrophysiological tests: Nerve conduction studies (NCSs) are the most frequently used diagnostic tools for DSPN and used for confirmation. The main feature of DSPN is reduced distal lower extremity sensory nerve action potential amplitudes [74]. In early cases, nerve conduction velocity studies are usually normal when only small-diameter fibers are damaged. Although NCSs have limited utility in diagnosing small-fiber neuropathy in the early period of neuropathy related to diabetes, it is a reliable procedure to exclude any other etiology (superimposed nerve entrapments or inflammatory demyelinating polyneuropathies, neuropathies with asymmetrical symptoms or involvement of the upper limbs etc.) $[52,75]$. Despite much study reports that the underlying process is axonal degeneration and/or demyelination, axonal degeneration is the primary process [76]. Follow-up changes in peroneal motor conduction velocity or sural sensory amplitude have often been used as primary progression measures in diabetic neuropathy trials because they are objective, repeatable, and continuously variable [52, 75-77].

Laboratory tests: Small fiber predominant 
neuropathy presenting with pain and dyesthesias in the feet is usually an early manifestation of the typical DSPN in diabetes. To show decreased intra-epidermal nerve fiber density by Laser-evoked potentials and skin biopsy is very helpful because the clinical examination and nerve conduction studies may be normal in the early period $[73,78,79]$. Histological analysis of tissue taken from the distal part of the leg, within sural area $10 \mathrm{~cm}$ above the lateral malleolus in cases of suspected DSPN shows degenerated smalldiameter sensory fibers. A low intraepidermal nerve fiber density below the fifth percentile is usually considered confirmatory for a diagnosis of early cases of DSPN [42, 43]. Screening laboratory tests providing the highest yield of abnormality are blood glucose, serum B12 with metabolites (methylmalonic acid with or without homocysteine) and serum protein immunofixation electrophoresis in the patients with DSPN. Metformin interferes with the absorption of cobalamin and may contribute to vitamin deficiency. Vitamin B12 deficiency should be certainly evaluated because it can lead to progressive deterioration of nerve tissue frequently misdiagnosed as diabetic peripheral neuropathy [80-82]. On the other hand, functional deficiency of vitamin B12 (ie, normal cobalamin but elevated MMA levels) is another problem with a tripling of neuropathy frequency in type 2 diabetes patients (regardless of metformin use) [80-82]. Infections (e.g., Lyme disease, HIV, Hepatitis), vasculitis, alcohol use, cancer and related paraneoplastic syndromes should also be assessed in order to exclude other etiologies of polyneuropathy. Furthermore, diabetic neuropathy may be associated with demyelination, and diabetes mellitus, multifocal motor neuropathy and CIDP may have elevated CSF protein [83]. Because of this diagnostic problem, we should consider all possible causes of neuropathy in order to properly treat the diabetes patients [48]. Genetic testing may be considered in patients only with a history of hereditary neuropathy and cryptogenic polyneuropathy who exhibit a hereditary neuropathy phenotype [84].

\section{B. Transient symmetric neuropathies (Acute, painful, sensory predominant)}

\section{Diabetic neuropathic cachexia (DNC)}

It is an uncommon syndrome and symmetric sensory peripheral neuropathy is associated with "weight loss up to $60 \%$ of total body weight, symmetric painful dysesthesias over the limbs and trunk which is typically affecting trunk" $[48,85,86]$.
It is not associated with weakness similar to DSPN. Patients may experience neuropathic pains tending to be worse at night or during periods of relaxation. The patients complain very severe pain, but sensory impairment associated with the DNC is usually minimal. The pain resolves with weight gain. Depression is one of the hallmarks of the syndrome [48]. The DNC is reversible with adequate diabetic control over weeks to months, unlike other symmetric diabetic neuropathies. Most reported patients are adult males, ordinarily in the sixth or seventh decades of lifetime. The DNC can occur in both types of diabetes patients and are associated with poor glucose control $[48,55,56]$.

\section{Hyperglycemic neuropathy}

It is likewise a secret and mostly transient state. Hyperglycemic neuropathy can ocur at the time of diagnosis or may follow an episode of ketotic coma, and the symptoms rapidly subside once the diabetes is controlled. Distally located sensory paresthesias and pain have been declared but not proved to be due to hyperglycemia in these patients. The relationship between hyperglycemia and neuropathy is still not definite because the neuropathy can regress in these patients despite poor glucose control [48].

\section{Insulin neuritis}

The insulin neuritis is usually characterized by symmetric neuropathy with acute onset of severe distal limb pain and autonomic dysfunction in relation to intensive glycemic control following the institution of insulin or intake of oral hypoglycemic agents (predominantly small-fiber neuropathy manifesting with pain and paresthesia) [48, 87-89]. Typical cases occur after several episodes of protracted hypoglycemia with mostly insulinoma [48]. A classic patient may complain of distal paresthesia similar to DSPN. Although insulin neuritis involves the upper extremities more than the lower extremities, footdrop is also common [48]. Neuropathic symptoms such as hyperalgesia and allodynia which are refractory to medications persist for weeks or months and regress after control of hypoglycemia [47, 48].

\section{Transient asymmetric neuropathies (Acute, painful, sensory-motor type)}

\section{Diabetic radiculoplexus neuropathies}

They are acute or subacute forms of neuropathy and presents commonly with abrupt onset of pain followed by weakness and primarily affect patients 
with mild diabetes [1]. The symptoms progress, then stabilizes and gradually improve over a few weeks. There are three types of radiculoplexus neuropathy named according to location.

a- Cervical radiculoplexus neuropathy: It is a very uncommon form of the radiculoplexus neuropathy.

b- Thoracic radiculoneuropathy: It typically presents with abrupt onset severe pain (burning, stabbing or belt like) with contact hyperesthesia along the thoracic spine, chest, rib cage or upper abdominal wall. Popping out of the abdominal wall may come because of muscle weakness. Truncal radiculoneuropathy can be symmetrical and involve multiple dermatomes, so it may be confused with intra-abdominal or thoracic disease or herpes zoster $[1,4,90]$. Needle EMG findings include fibrillations in the paraspinous or abdominal wall muscles [65].

3- Lumbar radiculoplexopathy: It causes the most morbidityand known by different names, including diabetic amyotrophy, Bruns-Garland syndrome, diabetic mononeuritis multiplex, diabetic polyradiculopathy, proximal diabetic neuropathy, and others $[1,90]$. Motor involvement is predominant and weakness and wasting in the thigh are seen after unilateral severe pain to be more severe at night in the anterior thigh, buttock, lumber area or knee. The distal muscles of the leg are rarely involved. Knee jerk is absent $[1,90]$.

\section{Diabetic mononeuritis multiplex}

Mononeuritis multiplex (MNM) is a syndrome of painful, unilateral or asymmetrical, asynchronous sensory and motor multiple neuropathies at least 2 separate nerve areas $[90,92]$. The areas affected by MNM depend on the underlying cause of the condition. This case of neuropathy tends to occur in older patients with relatively mild cases of diabetes. After the abrupt onset of pathology because of occlusion of the vasa nervosum in one nerve, multiple nerves are affected in a random distribution. As the condition worsens, it becomes less multifocal and more symmetrical. Individuals with diabetes typically present with acute onset of severe, unilateral thigh pain that is followed rapidly by weakness and atrophy of the anterior thigh muscles and loss of the knee reflex (see diabetic amyotrophy) $[1,90]$. As mononeuropathy multiplex or multifocal neuropathy can occur with diabetes mellitus, it can be also associated with many different conditions such as infections, rheumatological disorders, cancer-related conditions and hematological conditions. In electrodiagnostic studies, a reduction in the motor and sensory nerve action potential amplitudes and minimal alterations in nerve conduction velocity are seen in the patients with MNM [92]. In diabetic multifocal motor neuropathy, mild inflammatory infiltrates and loss of axons may be observed in addition to conduction bloks [48, 92]. The needle electrode examination findings can vary and acute and chronic denervation may be seen depending on the severity and time course of the disorder. Nerve biopsy may be required as part of the diagnostic evaluation in cases with vasculitis which is usually treatable with immunosuppressive medications [48]. Any condition with asymmetrical polyneuropathy is considered in the differential diagnosis of MNM. As last words, the usual causes need to be pursued (vasculitis, infections, and hereditary) if a diabetic patient develops a true MNM [65].

\section{Cranial neuropathy}

The upper cranial nerves are affected (third, sixth, rarely fourth and seventh in order of frequency) [48, 93]. Diabetic third nerve palsy presents acute ophthalmoplegia with abrupt onset of pain behind or above the eye, followed by double vision, unilateral ptosis, paresis of the oculomotor innervated muscles and sparing of the pupil which is the hallmark of diabetic third nevre palsy [48, 93]. Cranial neuropathy is attributed to presumed microvascular ischemia of nerve $[93,94]$. If there is pupillary involvement or absence of pain, there is concomitant autonomic nerve involvement or aneurysm which must be excluded by neuroimaging $[93,94]$. Typically, cranial neuropathies in diabetes mellitus improve and settle within 2 to 3 months $[48,93,94]$. Imaging studies may be required to rule out stroke in some shells. Nevertheless, history alone without additional testing is sufficient in the bulk of these patients [65]. Pupillomotor function impairment (e.g., decreased diameter of dark adapted pupil) and Argyll-Robertson pupil abnormality can be seen as a result of autonomic dysfunction [1].

\section{Mononeuropathies}

Mononeuropathies often emerge during periods of transition in the diabetic illness, or when there has been rapid weight loss and nerve entrapment seems to be more common than nerve infarction $[89,90]$. The nerves most frequently affected are the femoral, sciatic and peroneal ones in that order. Patients with diabetes are also at increased risk of entrapment 
mononeuropathy. A higher prevalence of compressive neuropathies of median or ulnar nerves exists in patients with diabetes in comparison to the general population [48]. The second most commonly seen neuropathy in the patients with diabetes is carpal tunnel syndrome, which is 3 times more common in comparison to normal population $[6,89,90]$. The mononeuropathies are easily can be diagnosed using electromyography.

\section{Diabetic autonomic neuropathy (DAN)}

All organs are susceptible to autonomic dysfunction because vasomotor, visceromotor, and sensory nerve fibers innervate every organ. DAN is a widespread disorder and may be manifested by dysfunction of one or more organ systems. DAN typically affects both parasympathetic and sympathetic parts of the autonomic nervous system, so it results in cardiovascular, gastrointestinal, urination, sweating, pupils, and metabolic disturbances [48, 95]. Clinical symptoms of DAN generally do not occur in early phases of diabetes and it manifests first in the vagus nerve which is the longest of the ANS nerves. The cardinal signs of DAN are orthostatic/postural hypotension, orthostatic bradycardia and orthostatic tachycardia, resting tachycardia, exercise intolerance, decreased hypoxiainduced respiratory drive, impaired heart rate variability, heart rate unresponsiveness to respiration and abnormal blood pressure regulation. [48, 96]. Because of all these problems mentioned above, DAN may be linked to increased incidence of asymptomatic ischemia, myocardial infarction, and decreased rate of survival after myocardial infarction [95, 96]. Age, duration of diabetes, obesity, smoking, and poor glycemic control are risk factors increasing DAN prevalence [96]. What a pity, most of the cases with DAN have subclinical forms without the presence of signs and symptoms and they are detectable only by tests $[48,96]$. Subclinical forms of DAN may be detected within 1 year of diagnosis with type 2 diabetes and within 2 years of diagnosis with type 1 diabetes [48, 96, 97]. Although cardiovascular autonomic neuropathy (CAN) is the most clinically important and easily-studied form of DAN, DAN involving other organ systems should also be viewed in the optimal care of patients with diabetes.

\section{Cardiovascular Autonomic Neuropathy (CAN)} There is a problem of autonomic control of the cardiovascular system in CAN and the earliest manifestations of autonomic neuropathy in diabetes tend to be associated with parasympathetic denervation because of vagal involvement which represents the longest fibers [48, 95]. Reduced heart rate variation is the earliest indicator of CAN and it increases mortality rate because of myocardial infarction or malignant arrhythmia [95, 97].

Symptoms and signs: Unexplained tachycardia or bradycardia, orthostatic hypotension and poor exercise tolerance should be assessed for the presence of CAN [48].

Tests: Nowadays it is possible to objectively identify early stages of CAN with the usage of different autonomic function tests. Screening tests should be performed particularly in the patients with DSPN, poor glycemic control, cardiovascular risk factors, and vascular diabetic complications [52]. In comparison of a variety of simple, validated, and noninvasive tests (e.g., Valsalva maneuver, Handgrip test, Heart rate response to deep breathing and standing, and blood pressure response to standing), valsalva maneuver and heart rate variation tests are more common than others [52, 95-97].

a- Exercise intolerance: When you begin to exercise, your heart rate increases rapidly to the level necessary to meet the metabolic needs of increased activity. Diabetic patients who are likely to have CAN show a reduced response in heart rate and blood pressure during exercise and it is called as exercise intolerance [95]. Degrees of exercise intolerance can vary and it can exhibit itself after the mildest exertions or require more sustained effort to be recognized. Fatigue, cyanosis, muscle cramps, insufficient heart rate, blood pressure changes and depression are the most common signs of exercise intolerance [95-96].

b- Orthostatic hypotension: Orthostatic hypotension is defined by consensus as a fall in blood pressure of at least $20 \mathrm{mmHg}$ systolic and/or 10 $\mathrm{mmHg}$ diastolic within three minutes in an upright position. Blood pressure and heart rate are measured after 5 min supine and at 1 and 3 min after standing; patients unable to stand may be assessed while sitting upright. Hypotension without a compensatory increase in heart rate $(<10$ beats/min) suggests autonomic impairment. Faintness, light-headedness, dizziness, confusion, or blurred vision with postural changes is seen. Symptoms occur within seconds to a few minutes of standing and resolve rapidly on lying down. Exercise or a heavy meal may exacerbate symptoms [96]. Questionnaires may be used to investigate orthostatic symptoms and their severity in 
dysautonomic conditions $[1,96]$.

c- Heart rate variability (HRV): Heart rate variability (HRV) is the physiological phenomen on of variation in the time interval between heartbeats. When the autonomic system is intact, heart rate increases in response to inspiration. Autonomic function can be evaluated with bedside cardiac monitoring. The autonomic evaluation consisted of measuring HRV for $30 \mathrm{~min}$ in supine position in absence of any physical, sensory, or pharmacological stimuli. R-R variation between supine and standing position is measured. If $\mathrm{R}-\mathrm{R}$ supine/R-R standing is lower than 1.03, it is accepted as abnormal [95, 98, 99]. Patients with abnormal R-R intervals or with autonomic symptoms or signs require further evaluation for diabetes $[95,98,99]$.

\section{Gastrointestinal Autonomic Neuropathy $(G A N)$}

It is difficult to evaluate gastrointestinal autonomic function in humans. Gastroparesis is the most debilitating complication of GAN $[1,99]$. The diagnosis of GAN is often one of exclusion, because many of the gastrointestinal symptoms related to sympathetic and parasympathetic nervous systems may be seen $(52,96]$. GAN symptoms are associatedwith poorglycemic control, malnutrition, abnormal postprandial regulation of blood pressure, poor quality of life, and a high rate of hospitalization in the patients $[52,100]$.

Symptoms and signs: Nausea, bloating, dysphagia and chest pain secondary to esophageal dysmotility, constipation and abdominal pain secondary to gastroparesis diabeticorum, diarrhea, and fecal incontinence may be seen. Up to $75 \%$ of patients with diabetes can experience these symptoms mentioned [100].

Tests: Objective gastric emptying measurement may be used for the diagnosis of gastroparesis. To quantify gastrointestinal symptoms, the Diabetes Bowel Symptom Questionnaire is applicable [52].

\section{Erectile Dysfunction}

Erectile dysfunction or impotence is the inability to develop or maintain an erection of the penis during sexual activity. The most important organic causes are aging ( $>60$ years of age), cardiovascular disease and diabetes. Adverse or unanticipated drug interactions, obesity, sex (hypogonadism and prolactinoma), medical causes (groin hernia, trauma from prostatectomy surgery, multiple sclerosis, kidney failure), drugs (SSRIs and nicotine), alcohol use, tobacco use and psychosocial causes (anxiety, mental disorders, stress) are potential risks [52]. Erectile dysfunction affects $30-40 \%$ of diabetic men and is an early marker of cardiovascular risk because of endothelial dysfunction [1]. The use of validated questionnaires such as the international index of erectile function or quality of erection questionnaire is the most appropriate method to evaluate the erectile dysfunction symptoms $[52,101,102]$. In practice, the problem is likely to be physiological if the patient never has an erection; it could be physiological or psychological if sometimes (however rarely). Duplex ultrasound exam, the bulbocavernosus reflex test, nocturnal penile tumescence assessment and cavernosography are the most commonly used investigations to determine erectile dysfunction. Specific testing may be recommended in patients not responding to phosphodiesterase- 5 inhibitor which is the first effective oral treatment available for erectile dysfunction [52]. In female with genitourinary autonomic neuropathy, loss of lubrication is seen [95, 99]. Retrograde ejaculation due to neuropathy of the bladder sphincter is another complication of diabetes [99].

\section{Diabetic Sudomotor Dysfunction}

Loss of thermoregulatory sweating in a 'glove and stocking'distribution that can extend to the upper parts of thelimbs and the anterior abdomen, conforms the length dependency of diabetic neuropathy. Not only anhidrosis but also hyperhidrosis can be seen as a sign of DAN [1, 103]. Abnormal production of sweatthat appears over the upper part of the body after consumption of even non-spicy foods, occurs in occasionalcases [103]. The quantitative sudomotor axon reflex test is capable of detecting distal smallfiber polyneuropathy with a sensitivity of $75 \%$ [52].

\section{Diabetic Bladder Dysfunction}

In patients with diabetes and bladder dysfunction, sensory and autonomic nerve fibers areaffected [104]. Patients often remain asymptomatic in early stagesand impaired bladder sensation is usually the first manifestation of lower urinary tract involvement [1]. The usual symptoms are straining, hesitation and weakness of stream $[52,104]$. Impaired sensation of bladder fullness leads to overstretched bladder, reduced bladder contractility, increased residual urine and impaireduroflow in the patients with diabetic bladder dysfunction [104]. 


\section{Conclusion}

DSPN and carpal tunnel syndrome are commonly experienced problems in the patients with diabetes. Clinical evaluation and use of scoring systems for the evaluation of diabetic polyneuropathy is an important step to get a correct diagnosis. NCSs confirm the diagnosis of DSPN, entrapment neuropathies, MNM or radiculoplexopathy. SWME (1-10 g), vibrating test and ankle reflex can be used confidently for annual screening of diabetic neuropathy in diabetic patients. By using both the SWME and vibration tests, it can be differentiated non-diabetic control subjects from subjects with diabetes, as well as subjects with diabetes with and without neuropathy. The DN4 questionnaire is utilized for the evaluation of neuropathic pain. The primary advantage of this screening instrument which is comfortable to use and delivers a good diagnostic performance is to identify potential patients with neuropathy, especially by nonspecialists. Because neuropathy at the stage in which only small characters are affected can be annulled, it is important to diagnose DSPN in early phases. Skin biopsy taken from the dermatomal area of the sural nerve, laser-doppler-imager flare technique, corneal confocal microscopyare used to assess small fiber dysfunction in these patients with peripheral neuropathy, with the aim to demonstrate specific findings for diagnosing small fiber neuropathy.

\section{Conflict of interest}

The author disclosed no conflict of interest during the preparation or publication of this manuscript.

\section{Financing}

The author disclosed that they did not receive any grant during conduction or writing of this study.

\section{References}

[1] Deli G, Bosnyak E, Pusch G, Komoly S, Feher G. Diabetic neuropathies: diagnosis and management. Neuroendocrinology 2013;98:267-80.

[2] Thomas PK. Classification, differential diagnosis and staging of diabetic peripheral neuropathy. Diabetes 1997;46:54-7.

[3] Boulton AJ, Vinik AI, Arezzo JC, Bril V, Feldman EL, Freeman R, et al.; American Diabetes Association. Diabetic neuropathies: a statement by the American Diabetes Association. Diabetes Care 2005;28:956-62.

[4] Gupta A, Gupta Y. Diabetic neuropathy: Part 1. J Pak Med Assoc 2014;64:714-8.

[5] Dyck PJ, Kratz KM, Karnes JL, Litchy WJ, Klein R, Pach JM, et al. The prevalence by staged severity of various types of diabetic neuropathy, retinopathy, and nephropathy in a population-based cohort: the Rochester Diabetic Neuropathy Study. Neurology 1993;43:817-24.
[6] Pasnoor M, Dimachkie MM, Kluding P, Barohn RJ. Diabetic neuropathy part 1: overview and symmetric phenotypes. Neurol Clin 2013;31:425-45

[7] Tesfaye S, Boulton AJM, Dyck PJ, Freeman R, Horowitz M, Kempler P, et al.; Toronto Diabetic Neuropathy Expert Group. Diabetic Neuropathies: Update on Definitions, Diagnostic Criteria, Estimation of Severity, and Treatments. Diabetes Care 2010;33:2285-93.

[8] Dyck PJ, Davies JL, Clark VM, Litchy WJ, Dyck PJ, Klein CJ, et al. Modeling chronic glycemic exposure variables as correlates and predictors of microvascular complications of diabetes. Diabetes Care 2006;29:2282-8.

[9] Ali A, Iqbal F, Taj A, Iqbal Z, Amin MJ, Iqbal QZ. Prevalence of microvascular complications in newly diagnosed patients with type 2 diabetes. Pak J Med Sci 2013;29: 899-902.

[10] Wild S, Roglic G, Green A, Sicree R, King H. Global prevalence of diabetes: estimates for the year 2000 and projections for 2030 . Diabetes Care 2004;27:1047-53.

[11] Zilliox L, Russell JW. Treatment of diabetic sensory polyneuropathy. Curr Treat Options Neurol 2011;13:143-59.

[12] Smith AG, Russell J, Feldman EL, Goldstein J, Peltier A, Smith S, et al. Lifestyle intervention for pre-diabetic neuropathy. Diabetes Care 2006;29:1294-9.

[13] Albers JW, Herman WH, Pop-Busui R, Martin CL, Cleary P, Waberski B; Diabetes Control and Complications Trial (DCCT)/Epidemiology of Diabetes Intervention and Complications (EDIC) Research Group. Subclinical neuropathy among Diabetes Control and Complications Trial participants without diagnosable neuropathy at trial completion: possible predictors of incident neuropathy? Diabetes Care 2007;30:2613-8.

[14] Treede RD, Jensen TS, Campbell JN, Cruccu G, Dostrovsky JO, Griffin JW, et al. Neuropathic pain: redefinition and a grading system for clinical and research purposes. Neurology 2008;70:1630-5.

[15] Tesfaye S, Selvarajah D. Advances in the epidemiology, pathogenesis and management of diabetic peripheral neuropathy. Diabetes Metab Res Rev 2012;28:8-14.

[16] Bril V, Perkins BA. Validation of the Toronto Clinical Scoring System for diabetic polyneuropathy. Diabetes Care 2002;25:2048-52.

[17] Meijer JWG, van Sonderen E, Blaauwwiekel EE, Smit AJ, Groothoff JW, Eisma WH, et al. Diabetic neuropathy examination: A hierarchical scoring system to diagnose distal polyneuropathy in diabetes. Diabetes Care 2000;23:750-3.

[18] Meijer JW, Smit AJ, Sonderen EV, Groothoff JW, Eisma WH, Links TP. Symptom scoring systems to diagnose distal polyneuropathy in diabetes: The Diabetic Neuropathy Symptom score. Diabet Med 2002; 19:962-5.

[19] Shehab DK, Al-Jarallah KF, Abraham M, Mojiminiyi OA, AlMohamedy H, Abdella NA. Back to basics: ankle reflex in the evaluation of peripheral neuropathy in type 2 diabetes mellitus. QJM 2012;105:31520.

[20] Vrancken AF, Kalmijn S, Brugman F, Rinkel GJ, Notermans NC. The meaning of distal sensory loss and absent ankle reflexes in relation to age: a meta-analysis. J Neurol 2006;253:578-89.

[21] Mold JW, Vesely SK, Keyl BA, Schenk JB, Roberts M. The prevalence, predictors, and consequences of peripheral sensory neuropathy in older patients. J Am Board Fam Pract 2004;17:309-18.

[22] Siao P, Cros DP. Quantitative sensory testing. Phys Med Rehabil Clin N Am 2003;14:261-86.

[23] Perkins BA, Olaleye D, Zinman B, Bril V. Simple screening tests for peripheral neuropathy in the diabetes clinic. Diabetes Care 2001;24:250-6.

[24] Krishnan STM, Quattrini C, Jeziorska M, Malik RA, Rayman G. Abnormal LDIflare but Normal Quantitative Sensory Testing and Dermal Nerve Fiber Density in Patients with Painful Diabetic Neuropathy. Diabetes Care 2009;32:451-5.

[25] Feng Y, Schlösser FJ, Sumpio BE. The Semmes Weinstein monofilament examination is a significant predictor of the risk of foot ulceration and amputation in patients with diabetes mellitus. J Vasc Surg 2011;53:220-6.

[26] Feng Y, Schlösser FJ, Sumpio BE. The Semmes Weinstein 
monofilament examination as a screening tool for diabetic peripheral neuropathy. J Vasc Surg 2009;50:675-82.

[27] Cheng WY, Jiang YD, Chuang LM, Huang CN, Heng LT, Wu HP, et al. Quantitative sensory testing and risk factors of diabetic sensory neuropathy. J Neurol 1999;246:394-8.

[28] Smieja M, Hunt DL, Edelman D, Etchells E, Cornuz J, Simel D L.; for the International Cooperative Group for Clinical Examination Research. Clinical examination for the detection of protective sensation in the feet of diabetic patients. J Gen Intern Med 1999;14:418-24.

[29] Blankenburg M, Boekens H, Hechler T, Maier C, Krumova E, Scherens A, et al. Reference values for quantitative sensory testing in children and adolescents: developmental and gender differences of somatosensory perception. Pain 2010;149:76-88.

[30] Davis EA, Jones TW, Walsh P, Byrne GC. The use of biothesiometry to detect neuropathy in children and adolescents with IDDM. Diabetes Care 1997;20:1448-53.

[31] Hirschfeld G, von Glischinski M, Blankenburg M, Zernikow B. Screening for peripheral neuropathies in children with diabetes: a systematic review. Pediatrics 2014;133:1324-30.

[32] Kaku M, Vinik A, Simpson DM. Pathways in the diagnosis and management of diabetic polyneuropathy. Curr Diab Rep 2015;15:35.

[33] Oyer D, Saxon D, Shah A. Quantitative assessment of diabetic peripheral neuropathy with use of the clanging tuning fork test. Endocr Pract 2007;13:5-10.

[34] Rolke R, Baron R, Maier C, Tölle TR, Treede RD, Beyer A, et al. Quantitative sensory testing in the German Research Network on Neuropathic Pain (DFNS): standardized protocol and reference values. Pain 2006;123:231-43.

[35] Shukla G, Bhatia M, Behari M. Quantitative thermal sensory testing - value of testing for both cold and warm sensation detection in evaluation of small fiber neuropathy. Clin Neurol Neurosurg 2009; 107:486-90.

[36] Hoeijmakers JG, Faber CG, Lauria G, Merkies IS, Waxman SG. Small fibre neuropathies--advances in diagnosis, pathophysiology and management. Nat Rev Neurol 2012;8:369-79.

[37] Mueller D, Obermann M, Koeppen S, Kavuk I, Yoon MS, Sack F, et al .Electrically evoked nociceptive potentials for early detection of diabetic small-fiber neuropathy. Eur J Neurol 2010;17:834-41.

[38] Smieja M, Hunt DL, Edelman D, Etchells E, Cornuz J, Simel DL.; for the International Cooperative Group for Clinical Examination Research. Clinical examination for the detection of protective sensation in the feet of diabetic patients. J Gen Intern Med 1999;14:418-24.

[39] Jiang MS, Yuan Y, Gu ZX, Zhuang SL. Corneal confocal microscopy for assessment of diabetic peripheral neuropathy: a metaanalysis. Br J Ophthalmol 2016;100:9-14.

[40] Tavakoli M, Petropoulos IN, Malik RA. Assessing corneal nerve structure and function in diabetic neuropathy. Clin Exp Optom 2012;95:338-47.

[41] Devigili G, Tugnoli V, Penza P, Camozzi F, Lombardi R, Melli G, et al. The diagnostic criteria for small fibre neuropathy: from symptoms to neuropathology. Brain 2008;131:1912-25.

[42] Lauria G, Cornblath DR, Johansson O, McArthur JC, Mellgren SI, Nolano M, et al. EFNS guidelines on the use of skin biopsy in the diagnosis of peripheral neuropathy. Eur J Neurol 2005;12:747-58.

[43] Gibbons CH, Griffin JW, Polydefkis M, Bonyhay I, Brown A, Hauer PE, et al. The utility of skin biopsy for prediction of progression in suspected small fiber neuropathy. Neurology 2006;66:256-8.

[44] Sommer C, Lauria G. Skin biopsy in the management of peripheral neuropathy. Lancet Neurol 2007;6:632-42.

[45] Krishnan ST, Rayman G. The LDIflare: a novel test of C-fiber function demonstrates early neuropathy in type 2 diabetes. Diabetes Care 2004;27:2930-5.

[46] Votrubec M, Thong I. Neuropathic pain - a management update. Aust Fam Physician 2013;42:92-7.

[47] Spallone V, Morganti R, D'Amato C, Greco C, Cacciotti L, Marfia GA. Validation of DN4 as a screening tool for neuropathic pain in painful diabetic polyneuropathy. Diabet Med 2012;29:578-85.

[48] Russell JW, Zilliox L. Diabetic neuropathies. Continuum (Minneap Minn) 2014;20:1226-40.
[49] Barrett AM, Lucero MA, Le T, Robinson RL, Dworkin RH, Chappell AS. Epidemiology, public health burden, and treatment of diabetic peripheral neuropathic pain: a review. Pain Med 2007;8:50-62. [50] Kaku M, Vinik A, Simpson DM. Pathways in the diagnosis and management of diabetic polyneuropathy. Curr Diab Rep 2015;15:35.

[51] Sharma KR, Cross J, Farronay O, Ayyar DR, Shebert RT, Bradley WG. Demyelinating neuropathy in diabetes mellitus. Arch Neurol 2002;59:758-65.

[52]Tesfaye S, Boulton AJ, Dyck PJ, Freeman R, Horowitz M, Kempler P, et al. Toronto Diabetic Neuropathy Expert Group: Diabetic neuropathies: update on definitions, diagnostic criteria, estimation of severity, and treatments. Diabetes Care 2010;33:2285-93.

[53] Dyck PJ. Severity and staging of diabetic polyneuropathy. In: Gries FA, Cameron NE, Low PA, et al., eds. Textbook of Diabetic Neuropathy. Stuttgart, Germany: Thieme; 2003;170-5.

[54] Vinik AI, Mehrabyan A. Diabetic neuropathies. Med Clin North Am 2004;88:947-99.

[55] Hoeijmakers JG, Faber CG, Lauria G, Merkies IS, Waxman SG. Small fibre neuropathies--advances in diagnosis, pathophysiology and management. Nat Rev Neurol 2012;8:369-79.

[56] Singh N, Armstrong DG, Lipsky BA. Preventing foot ulcers in patients with diabetes. JAMA 2005;293:217-28.

[57] Edwards JL, Vincent AM, Cheng HT, Feldman EL. Diabetic neuropathy: mechanisms to management. Pharmacol Ther 2008;120:134.

[58] Tesfaye S. Advances in the management of diabetic peripheral neuropathy. Curr Opin Support Palliat Care 2009;3:136-43.

[59] Zilliox LA, Ruby SK, Singh S, Zhan M, Russell JW. Clinical neuropathy scales in neuropathy associated with impaired glucose tolerance. J Diabetes Complications 2015;29:372-7.

[60] Boulton AJ, Vinik AI, Arezzo JC, Bril V, Feldman EL, Freeman R, et al.; American Diabetes Association Diabetic neuropathies: a statement by the American Diabetes Association. Diabetes Care 2005;28:956-2.

[61] Hartemann A, Attal N, Bouhassira D, Dumont I, Gin H, Jeanne S, Said G, Richard JL; Working Group on the Diabetic Foot from the French-speaking Society of Diabetology: Painful diabetic neuropathy: diagnosis and management. Diabetes Metab 2011;37:377-88.

[62] Apfel SC, Asbury AK, Bril V, Burns TM, Campbell JN, Chalk CH, et al. Positive neuropathic sensory symptoms as endpoints in diabetic neuropathy trials. J Neurol Sci 2001;189:3-5.

[63] Papanas N, Vinik AI, Ziegler D. Neuropathy in prediabetes: does the clock start ticking early? Nat Rev Endocrinol 2011;7:682-90.

[64] Dyck PJ, Albers JW, Andersen H, Arezzo JC, Biessels GJ, Bril V, et al. Toronto Expert Panel on Diabetic Neuropathy. Diabetic polyneuropathies: update on research definition, diagnostic criteria and estimation of severity. Diabetes Metab Res Rev 2011;27:620-8.

[65] Pasnoor M, Dimachkie MM, Kluding P, Barohn RJ. Diabetic neuropathy part 1: overview and symmetric phenotypes. Neurol Clin 2013;31:425-45.

[66] Said G, Goulon-Goeau C, Slama G, Tchobroutsky G. Severe earlyonset polyneuropathy in insulin-dependent diabetes mellitus. A clinical pathological study. N Engl J Med. 1992;326:1257-63.

[67] Stewart JD, McKelvey R, Durcan L, Carpenter S, Karpati G. Chronic inflammatory demyelinating polyneuropathy (CIDP) in diabetics. J Neurol Sci 1996;142:59-64.

[68] Uncini A, De Angelis MV, Di Muzio A, Callegarini C, Ciucci G, Antonini $\mathrm{G}$, et al. Chronic inflammatory demyelinating polyneuropathy in diabetics: motor conductions are important in the differential diagnosis with diabetic polyneuropathy. Clin Neurophys 1999;110:705-11.

[69] Jaradeh SS, Prieto TE, Lobeck LJ. Progressive polyradiculoneuropathy in diabetes: correlation of variables and clinical outcome after immunotherapy. J Neurol Neurosurg Psychiatry 1999;67:607-12.

[70] Singleton JR, Bixby B, Russell JW, Feldman EL, Peltier A, Goldstein J, et al. The Utah Early Neuropathy Scale: a sensitive clinical scale for early sensory predominant neuropathy. J Peripher Nerv Syst 2008;13:218-27.

[71] Dyck PJ, Davies JL, Litchy WJ, O'Brien PC. Longitudinal assessment of diabetic polyneuropathy using a composite score in the 
Rochester Diabetic Neuropathy Study cohort. Neurology 1997;49:22939.

[72] Xiong Q, Lu B, Ye H, Wu X, Zhang T, Li Y. The Diagnostic Value of Neuropathy Symptom and Change Score, Neuropathy Impairment Score and Michigan Neuropathy Screening Instrument for Diabetic Peripheral Neuropathy. Eur Neurol 2015;74:323-7.

[73] Cruccu G, Sommer C, Anand P, Attal N, Baron R, Garcia-Larrea L, et al. EFNS guidelines on neuropathic pain assessment: revised 2009. Eur J Neurol 2010;17:1010-8.

[74] Smith AG, Singleton JR. Diabetic neuropathy. Continuum (Minneap Minn) 2012;18:60-84.

[75] Hartemann A, Attal N, Bouhassira D, Dumont I, Gin H, Jeanne S, et al.; Working Group on the Diabetic Foot from the French-speaking Society of Diabetology: Painful diabetic neuropathy: diagnosis and management. Diabetes Metab 2011;37:377-88.

[76] Dyck PJ, Lais A, Karnes JL, O'Brien P, Rizza R. Fiber loss is primary and multifocal in sural nerves in diabetic polyneuropathy. Ann Neurol 1986;19:425-39.

[77] Greene DA, Stevens MJ, Feldman EL. Diabetic neuropathy: scope of the syndrome. Am J Med 1999;107(2B):2-8.

[78] Tavee J, Zhou L. Small fiber neuropathy: a burning problem. Cleve Clin J Med 2009;76:297-305.

[79] Devigili G, Tugnoli V, Penza P, Camozzi F, Lombardi R, Melli G, et al. The diagnostic criteria for small fibre neuropathy: from symptoms to neuropathology. Brain 2008;131:1912-25.

[80] Solomon LR. Diabetes as a cause of clinically significant functional cobalamin deficiency. Diabetes Care 2011;34:1077-80.

[81] Bell DS. Metformin-induced vitamin B12 deficiency presenting as a peripheral neuropathy. South Med J 2010;103:265-7.

[82] Leishear K, Boudreau RM, Studenski SA, Ferrucci L, Rosano C, de Rekeneire N, et al. Relationship between vitamin B12 and sensory and motor peripheral nerve function in older adults. J Am Geriatr Soc 2012;60:1057-63.

[83] Young RJ, Ewing DJ, Clarke BF. Chronic and remitting painful diabetic polyneuropathy. Correlations with clinical features and subsequent changes in neurophysiology. Diabetes Care 1988;11:34-40. [84] England JD, Gronseth GS, Franklin G, Carter GT, Kinsella LJ, Cohen JA, et al. Practice Parameter: evaluation of distal symmetric polyneuropathy: role of laboratory and genetic testing (an evidencebased review). Report of the American Academy of Neurology, American Association of Neuromuscular and Electrodiagnostic Medicine, and American Academy of Physical Medicine and Rehabilitation. Neurology 2009;72:185-92.

[85] D'Costa DF, Price DE, Burden AC. Diabetic neuropathic cachexia associated with malabsorption. Diabetic Med 1992;9:203-5.

[86] Wright DL, Shah JH. Diabetic neuropathic cachexia and hypothyroidism in a woman. Mo Med 1987;84:143-5.

[87] Gibbons CH, Freeman R. Treatment-induced diabetic neuropathy: a reversible painful autonomic neuropathy. Ann Neurol 2010;67:534-
41.

[88] Llewelyn JG, Thomas PK, Fonseca V, King RH, Dandona P. Acute painful diabetic neuropathy precipitated by strict glycaemic control. Acta Neuropathol 1986;72:157-63.

[89] Bansal V, Kalita J, Misra UK. Diabetic neuropathy. Postgrad Med J 2006;82:95-100.

[90] Tracy JA, Dyck PJ. The spectrum of diabetic neuropathies. Phys Med Rehabil Clin N Am 2008;19:1-26.

[91] Kelkar P, Parry GJ. Mononeuritis multiplex in diabetes mellitus: evidence for underlying immune pathogenesis. J Neurol Neurosurg Psychiatry 2003;74:803-6.

[92] Misra UK, Kalita J, Nair PP. Diagnostic approach to peripheral neuropathy. Ann Indian Acad Neurol 2008;11:89-97.

[93] Said G. Focal and multifocal diabetic neuropathies. Arq Neuropsiquiatr 2007;65:1272-8.

[94] Keane JR. Third nerve palsy: analysis of 1400 personally-examined inpatients. Can J Neurol Sci 2010;37:662-70.

[95] Vinik AI, Erbas T. Diabetic autonomic neuropathy. Hand Clin Neurol 2013;117:279-94.

[96] Spallone V, Bellavere F, Scionti L, Maule S, Quadri R, Bax G, et al; Diabetic Neuropathy Study Group of the Italian Society of Diabetology: Recommendations for the use of cardiovascular tests in diagnosing diabetic autonomic neuropathy. Nutr Metab Cardiovasc Dis 2011;21:69-78.

[97] Ziegler D. Diabetic cardiovascular autonomic neuropathy: prognosis, diagnosis and treatment. Diabetes Metab Rev 1994;10:33983.

[98] De Souza NM, Vanderlei LCM, Garner DM. Risk evaluation of diabetes mellitus by relation of chaotic globals to HRV. Complexity 2015:20842092.

[99] Vinik AI, Maser RE, Mitchell BD, Freeman R. Diabetic autonomic neuropathy. Diabetes Care 2003;26:1553-79.

[100] Chandrasekharan B, Srinivasan S. Diabetes and the enteric nervous system. Neurogastroenterol Motil 2007;19:951-60.

[101] Rosen RC, Riley A, Wagner G, Osterloh IH, Kirkpatrick J, Mishra A. The international index of erectile function (IIEF): A multidimensional scale for assessment of erectile dysfunction. Urology 1997;49:822-30.

[102] Lowy M, Collins S, Bloch M, Gillman M, Lording D, Sutherland P, et al. Quality of Erection Questionnaire correlates: Change in erection quality with erectile function, hardness, and psychosocial measures in men treated with sildenafil for erectile dysfunction. J Sex Med 2007;4:83-92.

[103] Freeman R. Autonomic peripheral neuropathy. Lancet 2005;365:1259-70.

[104] Burakgazi AZ, Alsowaity B, Burakgazi ZA, Unal D, Kelly JJ. Bladder dysfunction in peripheral neuropathies. Muscle Nerve 2012;45:2-8. 